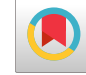

\title{
Primary Laryngeal Neuroendocrine Carcinoma: Two Cases of an Unusual Tumor in a Rare Location
}

\author{
Matin Ghazizadeh (iD ${ }^{1}$, Shervin Ghavami ${ }^{1}$ and Mahboobe Asadi (iD ${ }^{1, *}$ \\ ${ }^{1}$ Otorhinolaryngology Department, Shahid Beheshti University, Tehran, Iran \\ "Corresponding author: Otorhinolaryngology Department, Shahid Beheshti University, Tehran, Iran. Email: mahboobeasadi1986@gmail.com \\ Received 2018 July 26; Revised 2019 July 29; Accepted 2019 July 31.
}

\begin{abstract}
Introduction: Neuroendocrine neoplasm is an infrequent tumor in the larynx which can be sub-classified into five different types: typical carcinoid, atypical carcinoid, large cell carcinoma, small cell carcinoma, and paraganglioma. This tumor mostly occurs in smoker men. The common location of neuroendocrine tumor of the larynx is in the supraglottic area. Because of the complex nature of neuroendocrine neoplasms of the larynx, we found it so interesting to present. We emphasized the clinical importance and aggressive nature of this tumor despite its rarity.

Case Presentation: In the present study, we presented 2 cases of atypical carcinoid in non-smoker females. Case 1 was a 74-year-old non-smoker woman who was referred with a history of chronic dysphagia and left otalgia since one year ago. Laryngoscopy revealed a nonulcerated supraglottic epitheliomatous lesion. Biopsy showed atypical carcinoid. The patient had no lymphadenopathy or metastasis in the further workup. The second case was a 60-year-old non-smoker woman with a 4-month history of hoarseness, odynophagia accompanied by cervical lymphadenopathy. Laryngoscopy showed a smooth surface non-ulcerated transglottic tumor. Right vocal cord mobility was restricted.

Conclusions: Atypical carcinoid tumor is an underdiagnosed laryngeal malignancy. Although neuroendocrine tumor of the larynx is rare, physicians should be familiar with its unspecified presentations, pathology, clinical course, and prognosis.
\end{abstract}

Keywords: Laryngeal Neoplasm, Neuroendocrine Carcinoma, Atypical Carcinoid

\section{Introduction}

Although laryngeal neuroendocrine carcinomas are curious tumor they are the most common non-squamous tumors of this site. Neuroendocrine tumors are classified based on different histological categories such as paraganglioma with the neural origin and typical carcinoid, atypical carcinoid, small cell neuroendocrine carcinoma, and large cell neuroendocrine carcinoma with epithelial origin (1). About 700 neuroendocrine carcinomas of the larynx have been reported so far and its incidence has been estimated to be as low as $0.23 \%$ (2).

In the larynx, atypical carcinoid is the most common form of neuroendocrine carcinomas. It is frequently underdiagnosed (3). Although it is uncommon carcinoid, it is scientifically and clinically significant $(4,5)$. It usually presents with nonspecific upper respiratory tract symptoms and its diagnosis is based on histopathology and confirmed by immunohistochemical staining (6-8).

In this study, we presented 2 cases of atypical carcinoid and described their clinical presentation and aggressive disease course nature. The occurrence of this tumor in 2 non-smoker female patients with different clinical courses and difficulty in making an accurate diagnosis due to the complexity of neuroendocrine tumors classification are interesting features of this report.

\section{Case Presentation}

\subsection{Case 1}

A 74-year-old non-smoker woman referred to our otolaryngology clinic with a history of one-year progressive dysphagia and otalgia. She also complained of hoarseness and globous sensation in her throat since 2 months ago, without cough or aspiration. Her general condition was good. Direct laryngoscopy revealed a smooth nonulcerated tumor which was located in the supraglottis and involved the left false vocal cord and left arytenoids (Figure 1). It had a red and epitheliomatous appearance without necrosis. Both true vocal cords were mobile. The patient had no other symptoms. Her general physical examination was unremarkable, her skin was normal, and no enlarged cervical lymph nodes were palpated in the neck. 


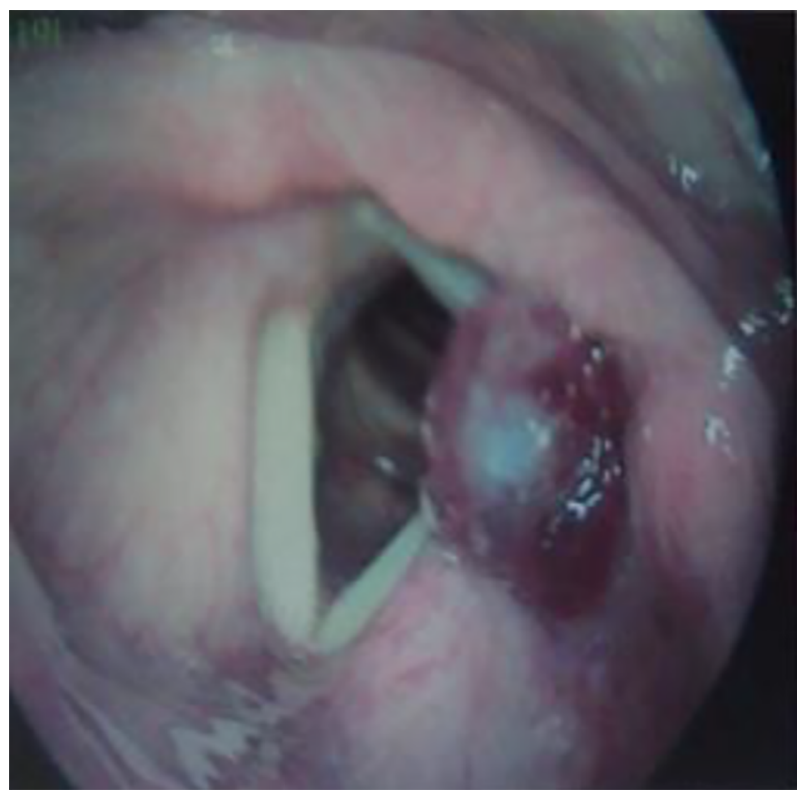

Figure 1. Laryngoscopic images showing a mass involving the left false vocal cord

The radiological investigation was requested and computerized tomography scan showed a supraglottic mass involving the left false vocal cord and left ventricle (Figure 2). Glottis and aryepiglottic folds were free of tumor and no metastatic cervical lymph node was detected. No distant metastatic lesion was noted on the chest, abdominal computerized tomography scan, and bone scan.

Direct laryngoscopy was performed under general anesthesia and excisional biopsy was taken and the tumor was precisely mapped. Microscopic histopathologic analysis showed a submucosal tumor with intact overlying mucosa, sheet-like and consisted of cells with moderate eosinophilic cytoplasm. In high-power field monomorphic cells with eosinophilic cytoplasm were seen. Prominent nucleoli and patchy necrosis were observed. These findings were consistent with the diagnosis of atypical carcinoid.

The diagnosis was confirmed by Immunohistochemistry which was positive for Chromogranin, Ki67, creatine kinase $\mathrm{AE} / 3$, and neuron-specific enolase (NSE).

The patient refused surgery, therefore, she was referred to the department of radiation oncology.

\subsection{Case 2}

The patient was a 60-year-old woman with hoarseness and dysphagia since 4 months ago. Her general condition was good. She had an enlarged lymph node in her lateral neck (level III). Direct laryngoscopy showed a smooth non-

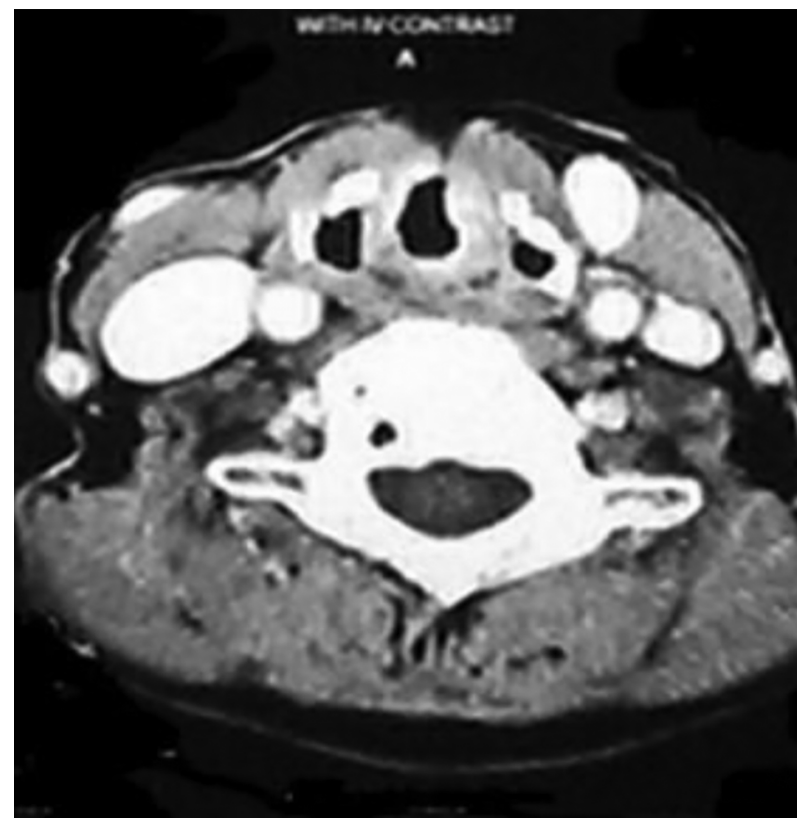

Figure 2. Contrast-enhanced computed tomography scan showing supraglottic tumor involves the left side.

ulcerated transglottic tumor. It was an epitheliomatous lesion in right true vocal cord with the anterior commissure extension. The mobility of the vocal cord was restricted to the right side. Her general physical examination was unremarkable. Her skin was normal.

The computerized tomography scan demonstrated a right transglottic mass involving the anterior commissure. Aryepiglottic folds were free. Some metastatic cervical lymph nodes were detected in level II. There were no metastatic lesions on the chest and abdominal in computerized tomography scan and bone scan.

Tumor mapping under general anesthesia was performed and the histopathologic findings were suggestive for neuroendocrine tumor. There was 2 - 10 mitosis per 10 high power field. Tumor cells with eosinophilic cytoplasm and mild to moderate atypical nuclei with prominent nucleoli the case was discussed among the panel of pathologists. Immunohistochemistry was advised and showed positive for chromogranin, neuron-specific enolase (NSE), synaptophysin, and ki67. The final diagnosis was atypical carcinoid.

The patient underwent total laryngectomy and right modified radical neck dissection.

Histopathological examination confirmed the atypical neuroendocrine tumor. Four out of six resected lymph nodes were involved by the tumor. The patient was referred to the department of radiation oncology. She re- 
ceived postoperative adjuvant chemo-radiotherapy.

After 4 months follow up, 2 neck masses were noted in level II $(5 \times 4 \mathrm{~cm})$ and level IV $(2 \times 2 \mathrm{~cm})$ which confirmed by computerized tomography scan.

She underwent bilateral radical neck dissection. Histopathological examination confirmed the previous diagnosis.

The patient was followed for 6 months without any problem.

\section{Discussion}

Neuroendocrine tumors are often recorded in the gastrointestinal tract or lungs, however head and neck are a rare site, the larynx is the commonest site in this anatomic region.

Although primary neuroendocrine carcinomas are the most common neoplasm of larynx in non-squamous category, its incidence is very low (9).

Neuroendocrine tumors of the larynx are classified as well-differentiated neuroendocrine carcinoma: typical carcinoid; moderately differentiated neuroendocrine carcinoma: atypical carcinoid tumor; poorly differentiated neuroendocrine carcinoma: large and small cell neuroendocrine carcinoma and Paragangliomas that are neuroendocrine tumors with neural origin $(2,4)$. However, some authors considered laryngeal large cell neuroendocrine carcinoma as a separate entity due to its aggressive behavior (6-10).

Typical carcinoids are very rare, while atypical carcinoid tumors are more frequent and had aggressive character (3). They usually metastasis to lymph nodes, bone, skin, liver, and lung $(7,11)$.

Atypical carcinoid is the most common neuroendocrine tumors of the larynx that represents $0.2 \%-0.6 \%$ of laryngeal malignancies $(3,5)$ laryngeal atypical carcinoid was first described by Goldman et al. in1969 (9).

Atypical carcinoid tumors of the larynx occur in heavy smokers in their 60s and the ratio in male to female is 3:1 (5). These tumors mostly occur in supraglottis (90\% of cases). The most common locations are aryepiglottic fold, arytenoid, and epiglottis (12). The presenting symptoms varied according to the location and compose of hoarseness, dysphagia, pharyngodynia, hemoptysis, and dyspnea (13).

Pathological features include nests of monomorphic cells with round nuclei and speckled chromatin, occasionally moderate atypia may be present (4). In neuroendocrine carcinomas, immunohistochemical study shows a positive result for chromogranin, synaptophysin, a cluster of differentiation (CD) 56, and neural cell adhesion molecule (NCAM) (14).
The recommended management is complete surgical removal with partial or total laryngectomy according to tumor size and extension. Since there is a high probability of cervical lymph nodes involvement, bilateral elective neck dissection should be performed. According to some references, limited dissection of level IIA and level III is enough for patients with no clinical lymphadenopathy. This tumor is reported to be resistant to both radiotherapy and chemotherapy. However, radiotherapy may be valuable if the tumor is locally extended or lymph node involvement is proved (2). Radiotherapy or chemotherapy is less effective and should be reserved for special situations (9). For atypical carcinoid tumors of the larynx, the 5-year survival rate reported being approximately $46.7 \%$ (11).

The clinical symptoms in our cases were not different from the usual presentations of neuroendocrine carcinomas of the larynx, but both cases were non-smoker females. The primary tumor locations were supraglottis as expected. The second case was more aggressive with extension to the glottis. An unusual finding in the first case was the indolent course of disease. She had a one-year history of the symptom without local extension or regional metastasis despite being an atypical carcinoid. Pathologic findings were typically being positive for Chromogranin, Ki67, creatine kinase $\mathrm{AE} / 3$, and neuron-specific enolase (NSE). Although distant metastasis is common in neuroendocrine tumors of the larynx, our cases had no distant metastasis.

\subsection{Conclusions}

A typical carcinoid tumor is an underdiagnosed malignancy. They can unexpectedly occur in none-smoker women. Although neuroendocrine tumor of the larynx is actually rare, physicians should be familiar with its unspecified presentations, pathology, clinical course, and prognosis.

\section{Acknowledgments}

None declared.

\section{Footnotes}

Conflict of Interests: There is not any conflict of interest among the authors.

Financial Disclosure: There was not any financial support.

Funding/Support: There was not any funding support. Patient Consent: Written informed consent was obtained from both patients. 


\section{References}

1. Ferlito A, Lewis JS Jr, Rinaldo A. The evolving management of laryngeal neuroendocrine carcinomas. Eur Arch Otorhinolaryngol. 2011;268(9):1247-8. doi: 10.1007/s00405-011-1666-z. [PubMed: 21681569].

2. van der Laan TP, Plaat BE, van der Laan BF, Halmos GB. Clinical recommendations on the treatment of neuroendocrine carcinoma of the larynx: A meta-analysis of 436 reported cases. Head Neck. 2015;37(5):707-15. doi: 10.1002/hed.23666. [PubMed: 24596175].

3. Mills SE. Neuroectodermal neoplasms of the head and neck with emphasis on neuroendocrine carcinomas. Mod Pathol.2002;15(3):264-78. doi: 10.1038/modpathol.3880522. [PubMed: 11904342].

4. Lewis JS Jr, Ferlito A, Gnepp DR, Rinaldo A, Devaney KO, Silver CE, et al. Terminology and classification of neuroendocrine neoplasms of the larynx. Laryngoscope. 2011;121(6):1187-93. doi: 10.1002/lary.21790. [PubMed: 21557244].

5. Ferlito A, Silver CE, Bradford CR, Rinaldo A. Neuroendocrine neoplasms of the larynx: An overview.Head Neck.2009;31(12):1634-46. doi: 10.1002/hed.21162. [PubMed:19536850].

6. Thompson ED, Stelow EB, Mills SE, Westra WH, Bishop JA. Large cell neuroendocrine carcinoma of the head and neck: A clinicopathologic series of 10 cases with an emphasis on HPV status. Am J Surg Pathol. 2016;40(4):471-8. doi: 10.1097/PAS.0000000000000580. [PubMed: 26735857]. [PubMed Central: PMC4792746].

7. McBride LC, Righi PD, Krakovitz PR. Case study of well-differentiated carcinoid tumor of the larynx and review of laryngeal neuroen- docrine tumors. Otolaryngol Head Neck Surg. 1999;120(4):536-9. doi: 10.1053/hn.1999.v120.a85070. [PubMed: 10187951].

8. Meacham R, Matrka L, Ozer E, Ozer HG, Wakely P, Shah M. Neuroendocrine carcinoma of the head and neck: A 20-year case series. Ear Nose Throat J. 2012;91(3):E20-4. doi: 10.1177/014556131209100317. [PubMed: 22430343].

9. Goldman NC, Hood CI, Singleton GT. Carcinoid of the larynx. Arch Otolaryngol.1969;90(1):64-7. doi:10.1001/archotol.1969.00770030066013. [PubMed: 5785991].

10. Dunn A, Broadfoot BG, Hunt J, Kaley JR, Atiq O, Gutta NB, et al Metastatic laryngeal large cell neuroendocrine carcinoma: A rare case of presentation and extreme tumor burden. J Cutan Pathol. 2018;45(3):229-33. doi: 10.1111/cup.13090. [PubMed: 29194700].

11. Soga J, Osaka M, Yakuwa Y. Laryngeal endocrinomas (carcinoids and relevant neoplasms): Analysis of 278 reported cases. J Exp Clin Cancer Res. 2002;21(1):5-13. [PubMed: 12071530].

12. Gillenwater A, Lewin J, Roberts D, El-Naggar A. Moderately differentiated neuroendocrine carcinoma (atypical carcinoid) of the larynx: A clinically aggressive tumor. Laryngoscope. 2005;115(7):1191-5. doi: 10.1097/01.MLG.0000166179.40750.1B. [PubMed:15995505].

13. Procopio G, Ricotta R, Fusi A, Celio L, De Dosso S, Catena L, et al. Neuroendocrine tumors of the larynx: A clinical report and literature review. Tumori. 2006;92(1):72-5. [PubMed: 16683387].

14. Bergeron M, Nadeau S. Surgery and radiotherapy for typical carcinoid of the nasopharynx: A case report. B-ENT. 2014;10(4):303-7. [PubMed: 25654955] 